\title{
Molecularly Imprinting Polymers (MIP) Based on Nitrogen Doped Carbon Dots and MIL-101(Fe) for Doxorubicin Hydrochloride Delivery
}

\author{
Yuqiong Shi ${ }^{1}$, Yuxuan Wang ${ }^{1}$, Jinhua Zhu ${ }^{1}, * \mathbb{D}$, Wei Liu ${ }^{1}$, Md. Zaved H. Khan ${ }^{2}$ and \\ Xiuhua Liu ${ }^{1, *}$ \\ 1 Henan International Joint Laboratory of Medicinal Plants Utilization, College of Chemistry and Chemical \\ Engineering, Henan University, Kaifeng 475004, China; sq13027537872@163.com (Y.S.); \\ wyx15224211156@163.com (Y.W.); 15290809123@163.com (W.L.) \\ 2 Department of Chemical Engineering, Jessore University of Science and Technology, \\ Jessore 7408, Bangladesh; zaved.khan@yahoo.com \\ * Correspondence: zhujinhua@henu.edu.cn (J.Z.); liuxiuhua@henu.edu.cn (X.L.); \\ Tel.: +86-371-23881589 (J.Z. \& X.L.)
}

Received: 28 July 2020; Accepted: 20 August 2020; Published: 23 August 2020

\begin{abstract}
MIL-based molecularly imprinted polymer (MIP) nanocomposites were successfully synthesized through a simple and versatile stirring auxiliary encapsulation method. MIP as a carrier has been applied to the highly efficient selective recognition and sustained release of doxorubicin hydrochloride (DOX). The adsorption mechanism and release behavior of MIP@DOX in vitro were also discussed. Adsorption studies showed that MIP using DOX as template had specific selectivity to DOX, and its optimal drug loading efficiency reached $97.99 \%$. The adsorption isotherm accorded with Freundlich models. The cumulative release curve showed that at the conditions of pH 5.5 and 7.4, the nanomaterials have a slow-release effect on the release of DOX. In addition, the cytotoxicity and bioactivity of MIP nanoparticles on HepG2 and HL-7702 cell lines measured by MTT assay also proved their low toxicity and biological activity. The cell activity of HepG2 and HL-7702 incubated with MIP for $24 \mathrm{~h}$ was $69.9 \%$ and $76.07 \%$, respectively. These results collectively illustrated that the MIP nano-materials synthesized in this study can be efficiently employed to the drug delivery systems.
\end{abstract}

Keywords: MIP; nanocomposites; drug delivery; DOX; controlled release

\section{Introduction}

Cancer is a main disease that jeopardizes human health and poses a critical threat to human life, and the incidence and mortality of cancer have been on the rise in recent years [1-3]. Doxorubicin hydrochloride (DOX) is an effective anticancer drug, which can remarkably inhibit the growth of cancer cells [4]. However, due to its toxicity to normal tissues and its inherent multidrug resistance effect, it is less appealing for direct application [5]. Therefore, many researchers are endeavoring to research and develop drug delivery systems (DDS) for DOX. For this purpose, polymer conjugates [6,7], metal-organic frameworks [8-10], and polymeric micelles [11] have been applied as carriers for DOX delivery.

Although the above carriers play a certain role in DOX transmission, these carriers generally have disadvantages such as relatively low drug loading rate and lack of specific recognition. One of the fascinating methods to solve these problems is the utilization of molecularly imprinted polymers (MIPs) such as DDS [12,13]. MIPs are synthetic receptors that can distinctively recognize target molecules in light of the shape, size and chemical function of corresponding binding sites [14-16]. Compared with other drug carriers, MIPs have the advantages of selective recognition, great stability, lower cost and 
uncomplicated synthesis. For example, André Luís MoraisRuela et al. [17] used nicotine and MAA (methacrylate) to synthesize a pH-MIP. The synthesized $\mathrm{pH}-\mathrm{MIP}$ was subjected to adsorption tested analytes at different $\mathrm{pH}$. The adsorption capacity was obviously higher when $\mathrm{pH} 6.5$ was applied. This was due to the protonation of the nicotine pyrrolidone ring and the carboxylate of the polymer, forming a strong electrostatic force which increased the adsorption of nicotine. At the same time, the author also conducted a comparative release experiment of MIP and none imprinted polymer (NIP) in an aqueous solution of $\mathrm{pH}$ 7.4. The results showed that MIP can release $45 \%$ of nicotine within $48 \mathrm{~h}$, which was higher than that of NIP (30\%), which is a good way to achieve the release of nicotine. Pawley et al. [18] used sensors to depict the drug release behaviors of nanoporous silver organic frameworks and introduced new applications of MIP-based thermal sensing platforms. The silver nanopore matrix applied acetylsalicylic acid (aspirin) as a model drug. The drug release behaviors were investigated by placing the nanomaterials in phosphate buffer for $48 \mathrm{~h}$ and determination the drug concentration regularly. Therefore, an acrylamide-based MIP was synthesized, which can detect aspirin in a specific and selective method.

Carbon dots (CDs) is a new groups of carbon materials, which have been proverbially used in many fields. CDs have the advantages of good biocompatibility, low toxicity, nice water solubility, simple functional modification and good chemical inertia $[19,20]$. The combination of CDs and metal organic framework (MOF) has been widely used. Gu et al. [21] reported a novel bimetallic zirconium hafnium metal organic framework (ZrHf-MOF) inlayed with quantities of CDs (referred as CDs@ZrHf-MOF), which exhibited intense fluorescence and abundant amino functionalization. The prepared CDs@ZrHf-MOF is able to be used as a scaffold to anchor the aptamer chains to mensurate human epidermal growth factor receptor-2 (HER2) and living HER2-overexpressed Michigan Cancer Foundation-7 (MCF-7) cells. The fundamental characterization revealed that these CDs were implanted into the inner cavities of the ZrHf-MOF film without changing the nanostructure, which resulted in nice biocompatibility, intense fluorescence, and eminent electrochemical activity of CDs@ZrHf-MOF. Chen et al. [22] was the first who proposed a simple synthetic route to exploit a new type of MOF-based fluorescence functionalized PCMs (phase change materials) aided by a fluorescence object and a thermal energy guest. Cr-MIL-101- $\mathrm{NH}_{2}$ served as a perfect compatible porous carrier, CQD (carbon quantum dot) as an excellent fluorescent active guest and stearic acid as an outstanding thermal energy guest. This distinctive structure can effectively prevent fluorescent quenching induced by conventional aggregation during operation.

Herein, a MOF-based MIP was developed for drug delivery (Scheme 1). MIL and nitrogen-doped $\mathrm{CDs}(\mathrm{N}-\mathrm{CD})$ were first compounded, and the successfully synthesized material was used as the framework, DOX as template, and tetraethyl orthosilicate (TEOS) as cross-linking agent to synthesize MIP. The as-synthesized N-CDs@MIL-101@MIP nanomaterials were used to sustainable delivery of DOX. Furthermore, the MTT experiment using human hepatocellular liver carcinoma cells (HepG2) and normal human liver cells (HL-7702) were conducted to test the biocompatibility of the synthesized DOX-loaded MIP. As a result, the MIP-synthesized in this study showed an excellent effect in the DDS and may become a potential drug carrier in cancer treatment. 


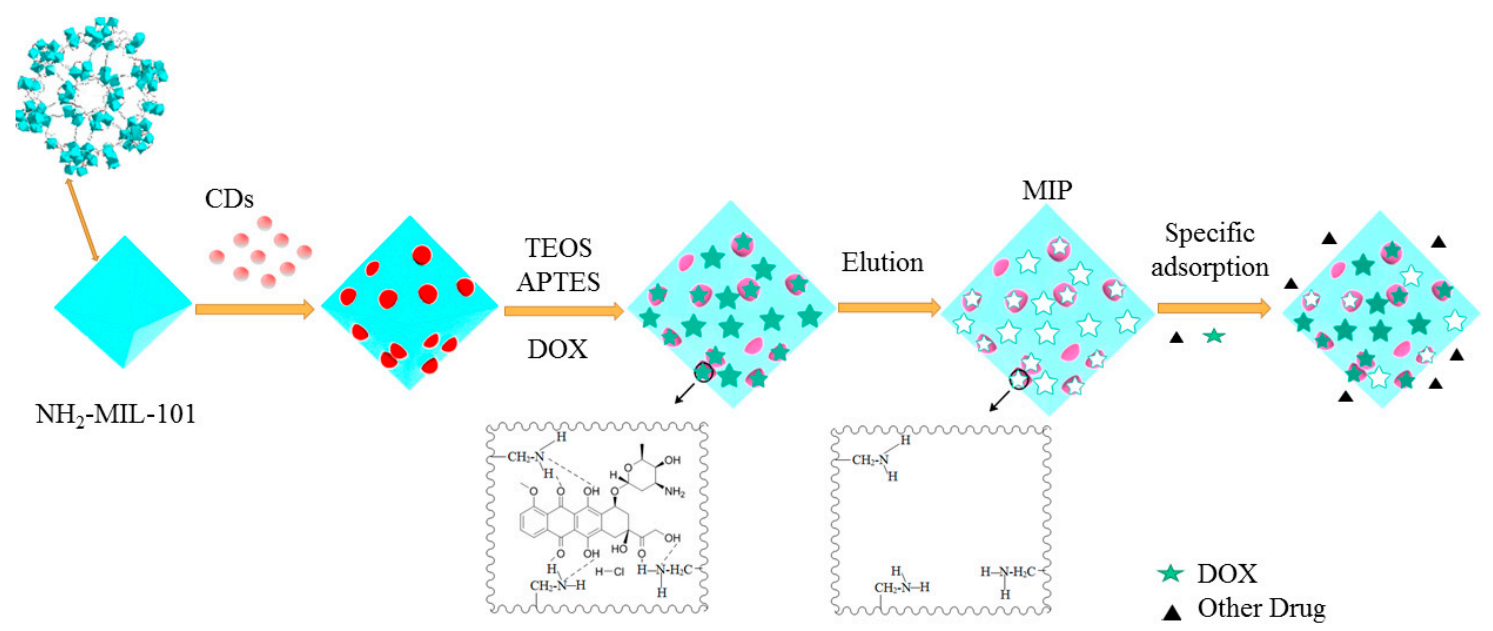

Scheme 1. Schematic diagram of the preparation and drug loading of MIP.

\section{Materials and Methods}

\subsection{Reagents and Apparatus}

Dioscorea opposita Thunb (Chinese yam) was obtained from Jiaozuo Baohetang Pharmacy Co., Ltd. (Jiaozuo, China). Ferric trichloride hexahydrate $\left(\mathrm{FeCl}_{3} \cdot 6 \mathrm{H}_{2} \mathrm{O}\right)$, 2-aminoterephthalic acid, 3-aminopropyl triethoxysilane (APTES), tetraethoxysilane (TEOS), doxorubicin (DOX), paclitaxel (PTX) and phosphate were purchased from Aladdin Biochemical Technology Co., Ltd (Shanghai, China). The organic solvents such as N,N-Dimethylformamide (DMF), acetate, methanol, ethanol were bought from Tianjin Deen Chemical Reagent Co., Ltd (Tianjin, China). Luteolin (LUT) and ethylenediamine were obtained from Chengdu MUST biotechnology Co., Ltd (Chengdu, China). Acetonitrile was obtained from Merck Drugs \& Biotechnology. The reagents used in the cell experiments are all from Zhengzhou Symus Biotechnology Co., Ltd (Zhengzhou, China). The 3-(4,5-dimethythia-zol-2-yl)-2,5-diphenylterazoliumbromide (MTT) was bought from Sigma Aldrich (Shanghai, China) Trading Co., Ltd (Shanghai, China). The HepG2 and HL-7702 cell lines were obtained from the Cell Bank of the Chinese Academy of Sciences (Shanghai, China). All other chemicals were analytical grade.

The shape of nanomaterials was characterized using GeminiSEM500 (Carl Zeiss Inc., Jena, Germany) scanning electron microscope (SEM). An infrared Fourier transform spectrometer (VERTEX 70, Bruker Inc., Karlsruhe, Germany) was used to obtain the FT-IR spectra. The fluorescence performance of the material was measured by a fluorescence spectrometer (F7000, Hitachi Limited., Tokyo, Japan). A TU-1900 spectrophotometer (Beijing Puxi General Instrument Co., Ltd, Beijing, China) was used to obtain the UV-vis absorption of drugs. A microplate reader (CLARIO star, BMG Labtech, Offenburg, Germany) was used for MTT assay.

\subsection{Preparation of $N$-Doped CDs (N-CDs)}

The N-doped CDs (N-CDs) was typically fabricated by one-pot hydrothermal process [23]. Specifically, $0.5 \mathrm{~g}$ powder of yam peel was added into $30 \mathrm{~mL}$ deionized water containing $25 \mu \mathrm{L}$ ethylenediamine. The mixed solution was transferred to a $100-\mathrm{mL}$ volumetric flask and heated at $180{ }^{\circ} \mathrm{C}$ for $15 \mathrm{~h}$. The obtained product was centrifuged at 10,000 rpm for 20 min to remove large particles and the supernatant was collected. The as-prepared N-CDs were stored at $4{ }^{\circ} \mathrm{C}$.

\subsection{Synthesis of N-CDs@MIL-101(Fe) (N-MIL)}

MIL was synthesized using hydrothermal method [24]. Briefly, $0.675 \mathrm{~g}(2.45 \mathrm{mmol})$ of $\mathrm{FeCl}_{3} \cdot 6 \mathrm{H}_{2} \mathrm{O}$ and $0.225 \mathrm{~g}(1.24 \mathrm{mmol})$ of 2-aminoterephthalic acid $\left(\mathrm{NH}_{2}-\mathrm{H}_{2} \mathrm{BDC}\right)$ were dissolved in $\mathrm{DMF}(30 \mathrm{~mL})$. After heating in a Teflon-lined auto-clave at $110{ }^{\circ} \mathrm{C}$ for $20 \mathrm{~h}$, the reaction products were re-collected by 
centrifugation and washed several times with DMF and ethanol, respectively. Finally, the obtained solid was dried at $150{ }^{\circ} \mathrm{C}$ for $8 \mathrm{~h}$.

A $0.100-\mathrm{g}$ amount of the above-dried MIL-101 powder was dissolved in $10 \mathrm{~mL}$ DMF, $5 \mathrm{~mL}$ N-CDs solution was slowly added. After agitating at room temperature for $2 \mathrm{~h}$, the products were collected and washed by ethanol and water for 3 times, respectively. Finally, the acquired solid (N-MIL) was dried at $80{ }^{\circ} \mathrm{C}$ for $8 \mathrm{~h}$ for following use.

\subsection{Synthesis of Template MIP Nanoparticles}

MIP nanomaterials were synthesized as follows [25]: $0.003 \mathrm{~g}$ of DOX as template was dissolved in acetonitrile $(3.0 \mathrm{~mL})$. After scattering of $0.050 \mathrm{~g} \mathrm{~N}$-MIL, APTES $(0.600 \mathrm{~mL})$ was introduced into the mixed solution, followed by stirring for $60 \mathrm{~min}$. Afterwards, cross-linker TEOS $(0.702 \mathrm{~mL})$ and catalyst (acetic acid, $1.0 \mathrm{~mL} 0.2 \mathrm{~mol} / \mathrm{L}$ ) were added. After agitating for $30 \mathrm{~min}$, the prepolymerization product was put in a closed container and incubated at $60^{\circ} \mathrm{C}$ for $20 \mathrm{~h}$. Then, by $300 \mathrm{~mL}$ of methanol/acetic acid, 9:1 $v / v$, the obtained MIP was washed to remove the template until DOX was not detected.

\subsection{Drug Loading Study}

DOX adsorbed on the MIP by specific binding sites. One milligram of the MIP or N-MIL was balanced in $3 \mathrm{~mL}$ of DOX solution $(350 \mu \mathrm{g} / \mathrm{mL})$ on the shaker oscillator at room temperature for $10 \mathrm{~h}$. Then, the solution was centrifuged, and using UV-vis spectrometry at $480 \mathrm{~nm}$, the adsorption capacity of DOX was determined by observing the absorbance of DOX solution before and after the addition of MIP. The DOX drug loading efficiency (DLE\%) of MIP was calculated by the following equation:

$$
\operatorname{DLE}(\%)=\frac{\text { weight of drug loaded in MIP }}{\text { weight of MIP used }} \times 100
$$

For LUT and PTX loading, it is the same procedures as above, just replace the DOX with LUT or PTX.

\subsection{Release In Vitro}

The in vitro drug release research of MIP@DOX was performed in two $\mathrm{pH}$ conditions ( $\mathrm{pH} 5.5$ and 7.4) at temperature of $37 \pm 0.5{ }^{\circ} \mathrm{C}$. In short, $1 \mathrm{mg}$ MIP@DOX was dispersed in the PBS buffer $(5 \mathrm{~mL})$ at $\mathrm{pH} 5.5$ or 7.4. These nanomaterials were transferred to the dialysis bag (MWCO 8000-14000, Beijing Solarbio Science \& Technology Co., Ltd, Beijing, China), and the dialysis bag was soaked in $100 \mathrm{~mL}$ PBS buffer solution with corresponding $\mathrm{pH}$, and vibrated at $37{ }^{\circ} \mathrm{C}$ in the dark. At scheduled time intervals, $3 \mathrm{~mL}$ of the buffer solution was taking away from the released solution for UV-Vis absorbance determination $(480 \mathrm{~nm})$, and an isometric fresh PBS was replenished for following tests. The release process of N-MIL@DOX is the same as MIP@DOX. The cumulative release efficiency was calculated as follows:

$$
\text { Cumulative release }(\%)=\frac{C_{\mathrm{n}} \times V_{0}+V_{i} \sum_{i=1}^{n-1} C_{i}}{m} \times 100
$$

where $C_{n}(\mu \mathrm{g} / \mathrm{mL})$ represents the concentration of DOX sampling at a specific time. $V_{0}$ and $V_{i}(\mathrm{~mL})$ are the total volume of the release medium and the sampling volume, respectively. $m(\mathrm{mg})$ is the mass of nanomaterials.

\subsection{In Vitro Cytotoxicity Test}

The vitro cytotoxicity investigations were carried out following the rules of the Declaration of Helsinki of 1975, revised in 2013. According to point 23 of this declaration, before undertaking the research, we have obtained an approval from the Ethics Committee of Biomedical Scientific Research of Henan University (HUSOM2020-037) on 20 March 2020. HepG2 cells (human hepatocellular carcinoma cell line) and HL-7702 (Human normal liver cell line) were maintained in DMEM (Dulbecco's modified 
eagle medium) supplemented with 10\% (v/v) FBS (Fetal bovine serum), $100 \mathrm{U} \cdot \mathrm{mL}^{-1}$ penicillin and $100 \mathrm{U} \cdot \mathrm{mL}^{-1}$ streptomycin under an atmosphere of humidified $5 \% \mathrm{CO}_{2}$ at $37^{\circ} \mathrm{C}$. Cells were dissociated by trypsinization and then passaged when they reached a density of $106 \mathrm{cell} \cdot \mathrm{mL}^{-1}$.

Cell viability was investigated by MTT (3-(4,5-dimethyl-2-thiazolyl)-2,5-diphenyl-2-H-tetrazolium bromide) assay in this work. Specifically, HepG2 or HL-7702 cells in logarithmic growth period were dissociated and seeded to 96 -well plates with $2 \times 10^{4}$ cells per well. Then, they were maintained at $5 \%$ $\mathrm{CO}_{2}$ and $37^{\circ} \mathrm{C}$ and for $24 \mathrm{~h}$. Subsequently, $20 \mu \mathrm{g} / \mathrm{mL}$ of N-MIL, N-MIL@DOX, MIP and MIP@DOX were added separately, followed by incubation for 4, 8, 16, 20 and $24 \mathrm{~h}$, respectively. Then, the culture medium was discarded and $1 \times$ PBS was used to wash the cells. Then, $200 \mu \mathrm{L}$ of MTT $(0.5 \mathrm{mg} / \mathrm{mL})$ was introduced and the cells were incubated for $4 \mathrm{~h}$. After that, $100 \mu \mathrm{L}$ of dimethyl sulfoxide (DMSO) was added to each well after careful removal of supernatants and then the 96-well plate was placed on an oscillator with gentle shaking to make the crystallized formazan sufficiently dissolved in DMSO. Ultimately, the absorbance of each well was detected by a microplate reader at $490 \mathrm{~nm}$.

Interventionary studies involving animals or humans, and other studies requiring ethical approval, must list the authority that provided approval and the corresponding ethical approval code.

\section{Results and Discussion}

\subsection{Structure Characterization}

The scanning electron microscope (SEM) images of the synthesized MIL and N-MIL were displayed in Figure 1a,b. It was apparent that both MIL and N-MIL nanomaterials had a uniform polyhedron structure. The morphologies of MIL and N-MIL were shown an octahedral structure, though N-MIL was filled with N-CDs. Figure 1c,d were the diagrams of MIP, it was easy to see that MIP was produced by the combination of N-MIL and cross-linking agent. The microstructure and compositional distribution of the MIP nanomaterials were further investigated by EDX (SEM coupled with energy dispersive X-ray) elemental mapping. An overlay of $\mathrm{O}, \mathrm{C}, \mathrm{N}$ and Fe EDX maps was shown in Figure 1e, indicating that Fe mainly located in the core of the material, and its surroundings were cross-linked with $\mathrm{N}$ and $\mathrm{O}$, while $\mathrm{C}$ can be observed from the surface (Figure 1e). These showed that MIP with N-MIL performance and simple synthesis method was successfully constructed.
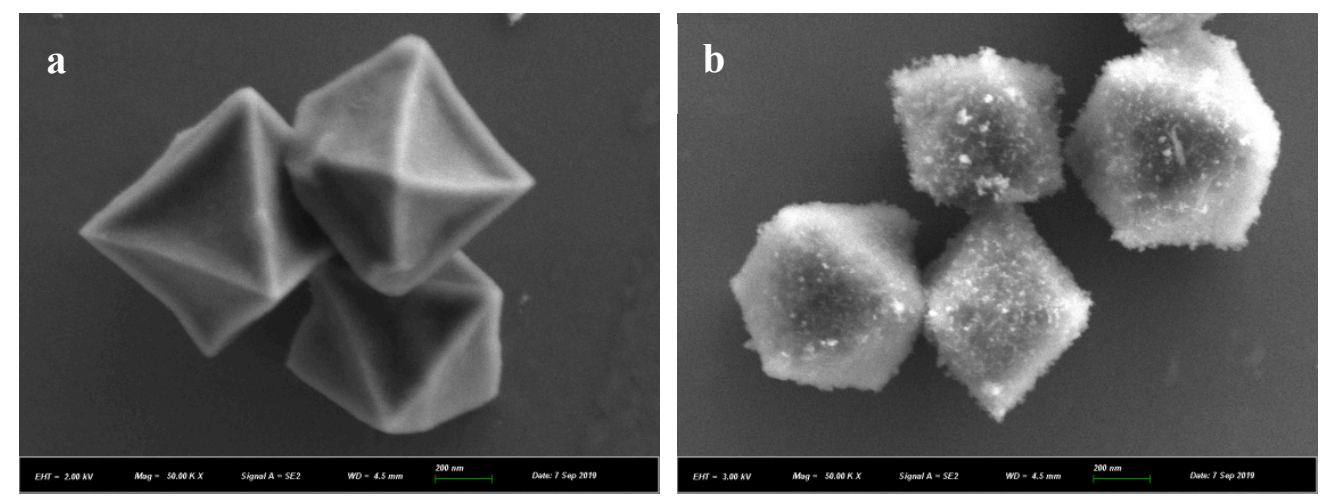

Figure 1. Cont. 

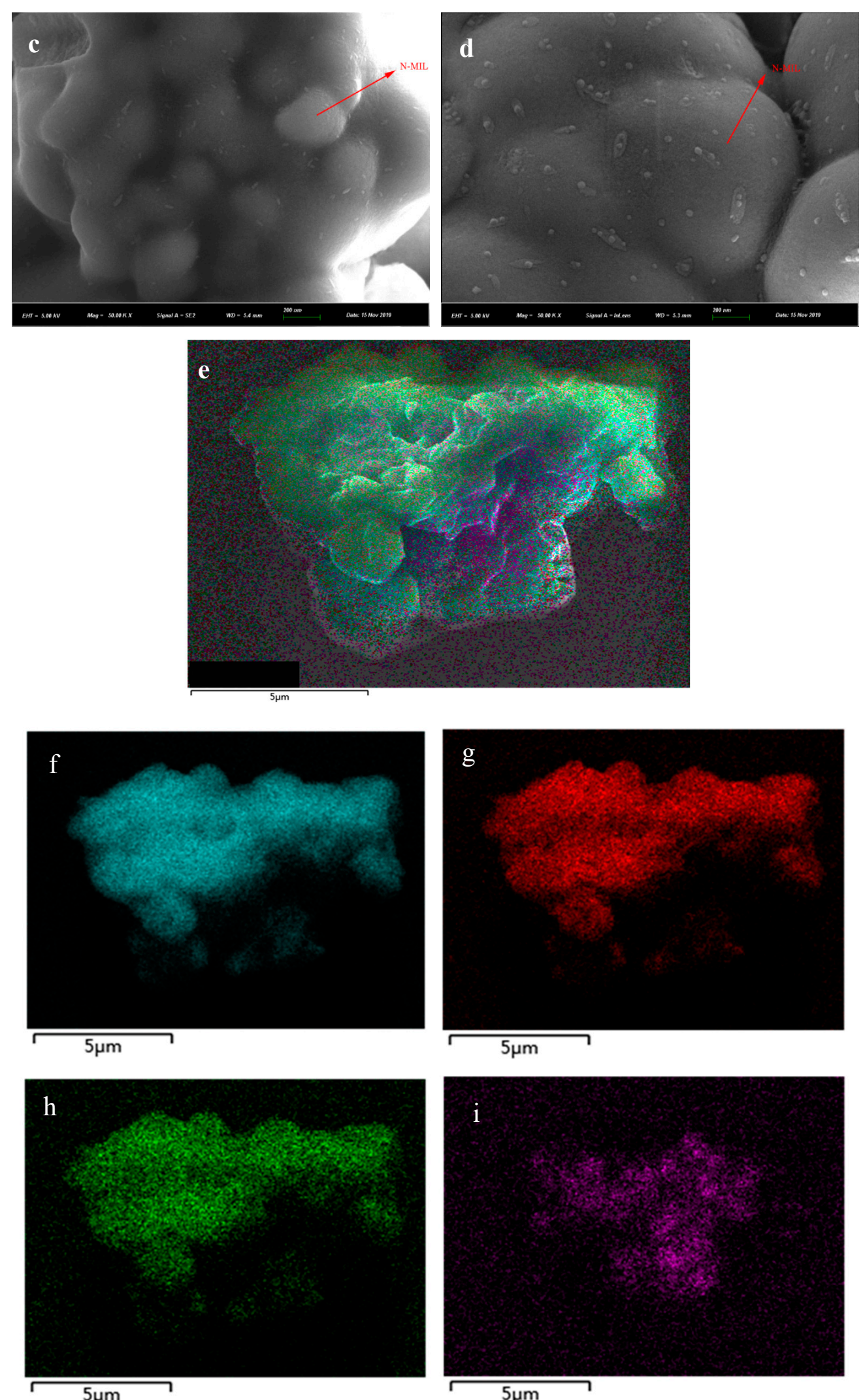

Figure 1. SEM image of MIL (a), N-MIL (b) and MIP (c,d). EDX elemental mapping of MIP (e). Blue is O element (f), red is $\mathrm{C}$ element $(\mathbf{g})$, green is $\mathrm{N}$ element $(\mathbf{h})$, and purple is Fe element (i). 
The Fourier transform infrared (FTIR) spectra of synthesized N-MIL, MIP and DOX were recorded in Figure 2. The absorbance peaks at $3430 \mathrm{~cm}^{-1}$ was attributed to the stretching vibration and bending vibration of $\mathrm{N}-\mathrm{H}$, which was considered to be on $\mathrm{NH}_{2}-\mathrm{MIL}$, separately [26]. The bands at $2930 \mathrm{~cm}^{-1}$ were attributed to the stretching vibration of $\mathrm{C}-\mathrm{H}$. The band at $1732 \mathrm{~cm}^{-1}$ was the stretching vibration of $\mathrm{C}=\mathrm{O}$. The band at $1640-1410 \mathrm{~cm}^{-1}$ was a typical band, which was ascribed to the stretching vibration of aromatic benzene rings [27]. Obviously, the appearance of typical bands of the MIP at $1640-1410 \mathrm{~cm}^{-1}$ may be attributed to MIL or residual DOX, demonstrating that the imprinted layer was successfully prepared [28]. In addition, the stretching vibrations features of Si-O-Si around $1100 \mathrm{~cm}^{-1}$ were observed [29], which indicated that silane coupling agents was hydrolyzed, thus verifying the successful encapsulation process of MIP on MIL-101.

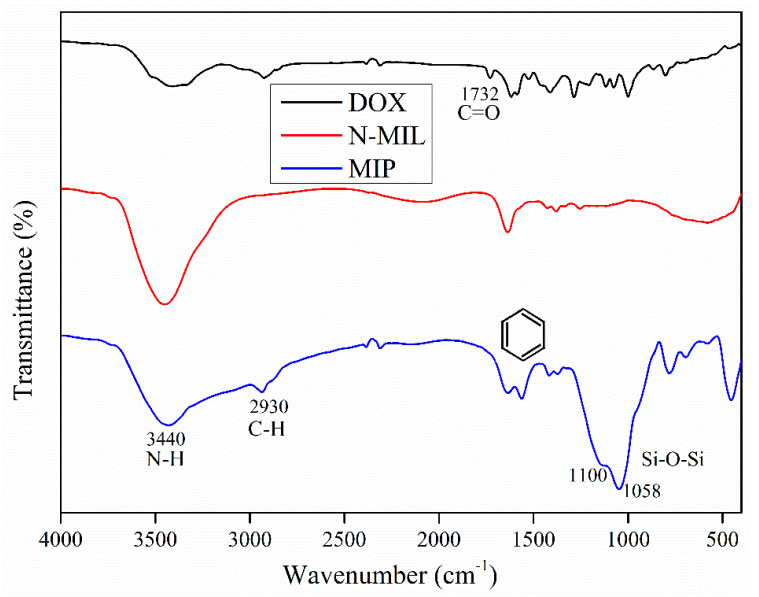

Figure 2. FT-IR spectra of DOX, N-MIL, MIP.

The fluorescence performance of the material was measured by a fluorescence spectrometer. As can be seen from Figure 3a, the maximum emission wavelength was $445 \mathrm{~nm}$ when the excitation wavelength was $345 \mathrm{~nm}$. As displayed in the inset of Figure 3b, the MIP solution (dissolved in water) was light brown. When irradiated with 365-nm ultraviolet light, it showed bright blue fluorescence. In addition, the fluorescence of MIP come from N-MIL encapsulated inside, and the emission peak position was almost no change compared with that of N-MIL, as shown in Figure $3 b$. The UV-vis absorption spectrum of DOX had a considerable overlap with the fluorescence emission spectrum of MIP, which indicated that this MIP could serve as a fluorescence donor to generate the energy resonance transfer effect (FRET) with DOX (Figure 3b) [30,31]. The fluorescence characteristics of MIP using DOX as template need to be further exploited for practical application.
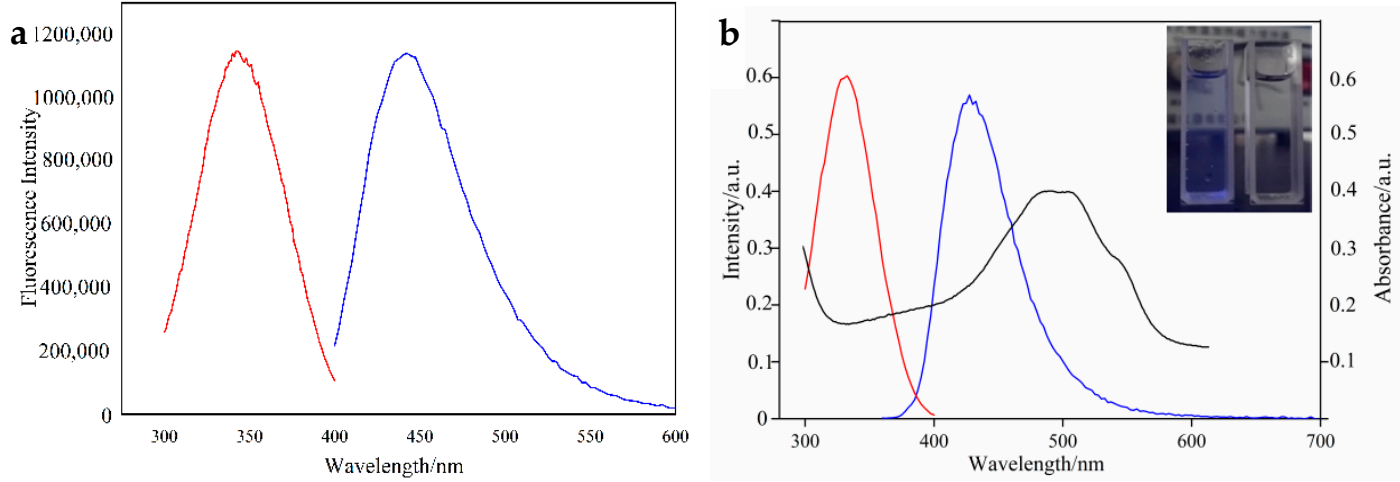

Figure 3. Fluorescence excitation spectrum (red line) and emission spectrum (blue line) of N-MIL (a) and MIP (b). The black line is the UV-vis absorption spectrum of DOX. Inset: Photographs of MIP under $365 \mathrm{~nm}$ UV lamp (left) and natural light (right) irradiation. 


\subsection{Specific Adsorption of MIP on Drugs}

The other two drugs (LUT and PTX) that have similar structures as DOX were used to study the specificity of MIP. As shown in Figure 4, comparing with N-MIL, MIP had absolutely specific recognition of DOX. The drug loading rate of MIP to DOX was approximately $100 \%$, while the loading rates of LUT and PTX on MIP were much lower. As showed in Scheme 1, the specificity of MIP to DOX may be due to the hydrogen bonds formed by the amines of the MIP particles (APTES) and the adjacent carbonyl and hydroxyl groups of the DOX. When DOX molecular first entered into the specific cavities of MIP particles, producing inductive fit with the MIP through hydrogen bonds described above. However, comparing with LUT, the higher drug loading rate of PTX on MIP was attributed to its structure being more adjacent carbonyl and hydroxyl groups that can form hydrogen bonds with the MIP. In addition, due to non-specific adsorption, N-MIL has a much lower drug loading capacity for all the three drugs, which also proved that only MIPs had the specificity and sensitivity for drug adsorption. As a result, MIP was synthesized with a certain specificity for the adsorption of DOX in this work.

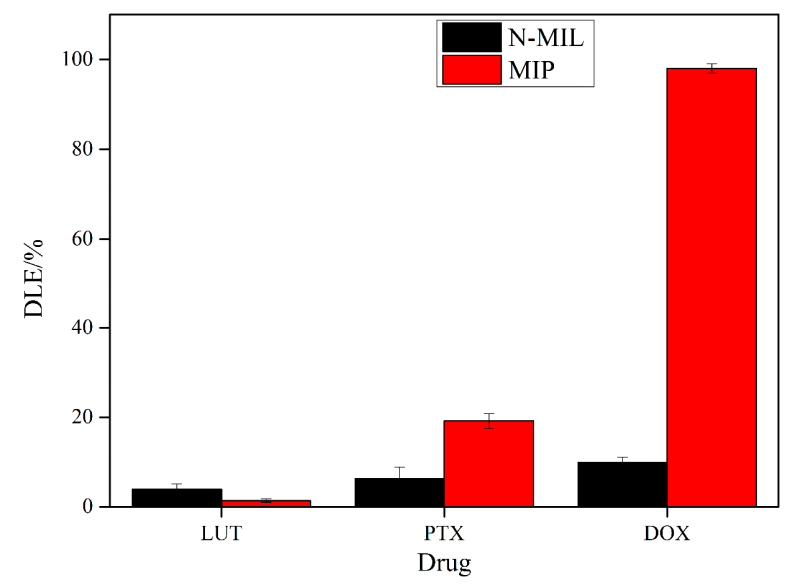

Figure 4. Specificity investigation of MIP. The concentration of LUT, PTX and DOX is 100, 100 and $350 \mu \mathrm{g} / \mathrm{mL}$, respectively.

\subsection{Drug Loading Studies}

In order to explore the adsorption mechanism, the influence of loading time and DOX concentration on the loading efficiency of MIP were investigated. For comparison purposes, the loading rate of DOX on N-MIL at same conditions was also researched. Totally, as depicted above, due to the specific recognition of MIP on DOX, the drug loading efficiency of MIP was much higher than that of N-MIL at each studied condition. Firstly, the drug loading time of DOX on MIP and N-MIL was studied using $1 \mathrm{mg}$ materials to adsorb $350 \mu \mathrm{g} / \mathrm{mL}$ DOX at room temperature. The results in Figure 5 a showed that the adsorption time had significant effect on the drug loading capacity of MIP while the loading time was less than $8 \mathrm{~h}$. When the adsorption time was more than $10 \mathrm{~h}$, the drug loading rate was almost unchanged. Further increasing the loading time, the adsorption rate instead decreased somewhat. We know that there is a balance between adsorption and desorption. After a certain period of time, the adsorption equilibrium reaches, further extending the adsorption time will make the drug that adsorbed on the surface partially desorbed, thus reducing the drug loading efficiency. The loading speed of DOX on MIP/N-MIL was relatively slow and, therefore, the loading process should be accomplished in $10 \mathrm{~h}$.

Moreover, in order to research the effect of DOX concentration on loading efficiency, different concentrations of DOX solution (i.e., 100, 150, 200, 250, 300, 350, and $400 \mu \mathrm{g} / \mathrm{mL}$ ) were added using $1 \mathrm{mg}$ MIP. As illustrated in Figure 5b, both drug loading efficiency on MIP and N-MIL increased when the concentration of DOX was increased from 100 to $350 \mu \mathrm{g} / \mathrm{mL}$ and then reached a plateau after $350 \mu \mathrm{g} / \mathrm{mL}$. It indicated that when the concentration of DOX was $350 \mu \mathrm{g} / \mathrm{mL}$, the drug loading 
efficiency of MIP reached to the maximum, which was nearly to $100 \%$, which was much higher than that of N-MIL (7.8\%), demonstrating the sensitivity and specificity of MIP to DOX adsorption. We also investigated the relationship between DLE and DOX concentration, and obtained the linear equation as follows: $D L E=0.27522 C_{\mathrm{dox}}+4.02076(\mathrm{n}=6, \mathrm{r}=0.9938)$. When the DLE was 50 , the concentration of DOX needed was $167 \mu \mathrm{g} / \mathrm{mL}$, while for N-MIL the maximum DLE was only 7.8\%, which also demonstrated the high sensitivity of MIP for DOX loading. The reason for the high DLE of MIP may be that when all the accessible specific cavities of MIP nanoparticles were saturated, the adsorption of analyte was generally attributed to nonspecific interactions, which can be the same for MIP and N-MIL nanoparticles. However, further increasing the concentration of DOX did not change the adsorption efficiency. The results demonstrated that MIP particles possess an identification ability for DOX that can be ascribed to the complementary cavities produced by DOX template. To summarize, the optimal conditions of drug concentration and reaction time were $350 \mu \mathrm{g} / \mathrm{mL}$ and $10 \mathrm{~h}$, respectively. Under the optimum conditions, the maximum amount of DOX adsorbed by MIP was $1029 \mu \mathrm{g} / \mathrm{mg}$, which accorded with the equilibrium absorption capacity (qe $1250 \mu \mathrm{g} / \mathrm{mg}$, data not shown). Compared to other reported DDS in Table 1, the drug loading content of the new materials in this work were better than that of the other drug delivery system.
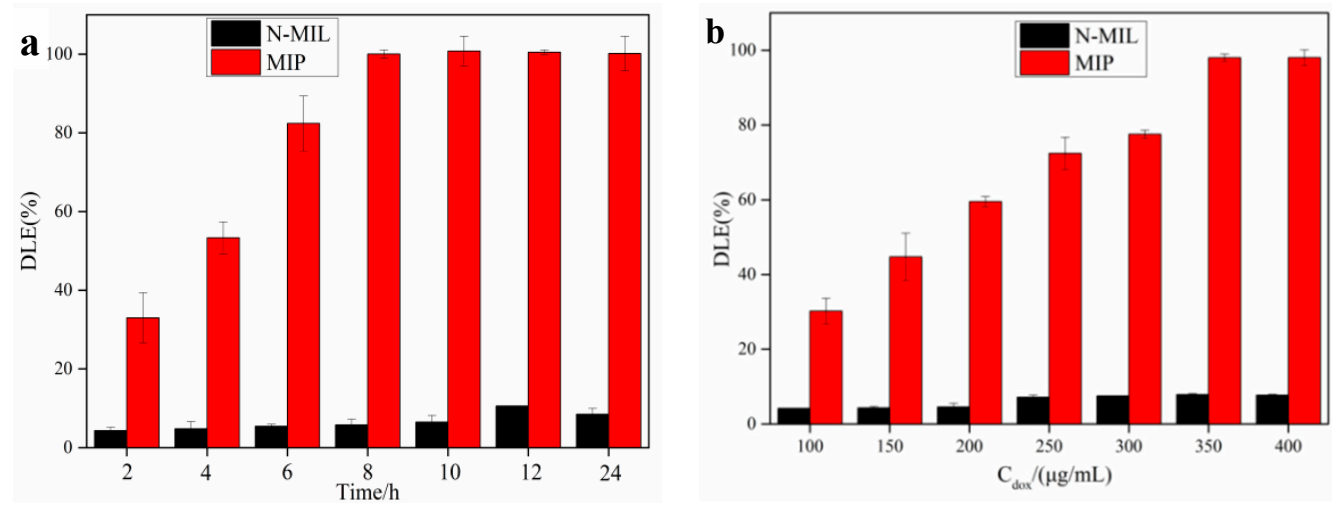

Figure 5. The effect of adsorption time (a) and drug concentration (b) on DOX loading.

Table 1. Comparison of the drug loading content of N-MIL/MIP with other reported drug delivery systems.

\begin{tabular}{cccc}
\hline Author & Drug Delivery System & Drug Loading Rate & Reference \\
\hline Jiang Lan & Amphiphilic polyrotaxane-based & $25.5 \%$ & {$[11]$} \\
Peng Huhong & block copolymer & $90 \%$ & {$[27]$} \\
Wang Haiyan & $\begin{array}{c}\text { Fluosescent double template } \\
\text { imprinted polymer }\end{array}$ & $11.05 \%$ & {$[28]$} \\
Shi Yuqiong & N-MIL-MIP & $97.9 \%$ & This work \\
\hline
\end{tabular}

The results of absorption research were analyzed by Freundlich and Langmuir isotherms to explore the best fitted model to describe the adsorption mechanism (Figure 6a,b), given by Equations (3) and (4) [32,33], respectively:

$$
\begin{gathered}
q_{e}=\frac{K_{L} Q_{m} C_{e}}{1+K_{L} C_{e}} \\
q_{e}=K_{F} C_{e}^{1 / n}
\end{gathered}
$$

where $q_{e}$ is adsorption capacity $(\mathrm{mg} / \mathrm{g})$ at equilibrium, $K_{L}$ is Langmuir constant $(\mathrm{L} / \mathrm{mg}), q_{m}$ is maximum monolayer adsorption capacity $(\mathrm{mg} / \mathrm{g}), K_{F}$ is Freundlich constant $(\mathrm{L} / \mathrm{g})$, and $\mathrm{n}$ is Freundlich exponent. 

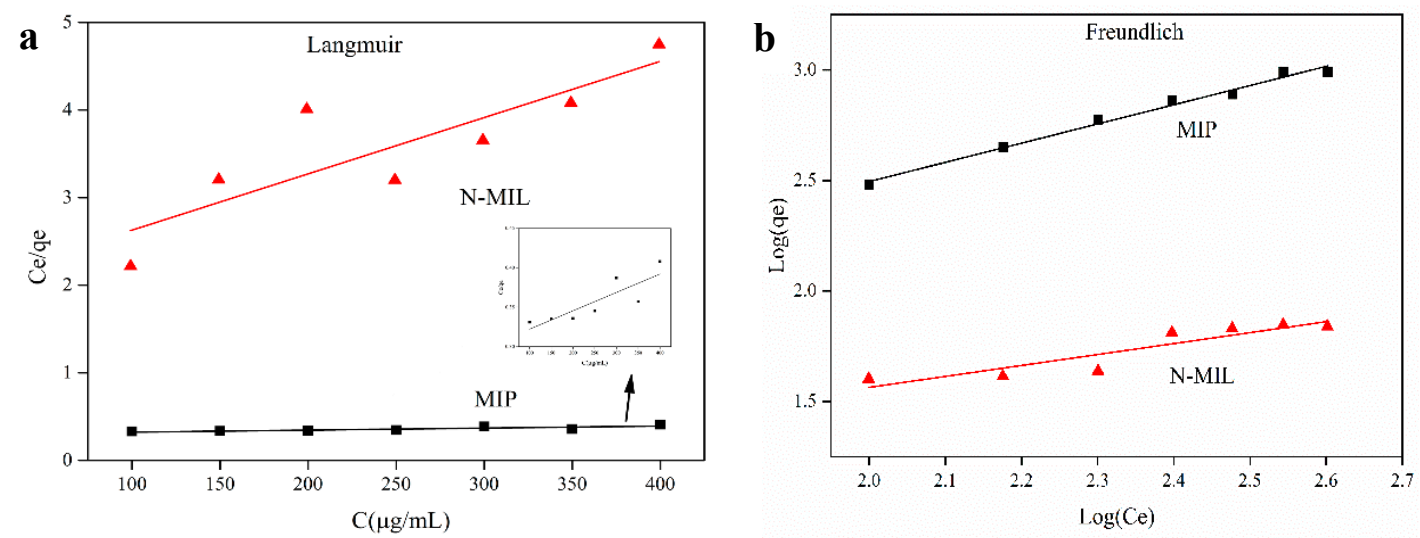

Figure 6. The adsorption isotherms fitted with Langmuir model (a) and Freundlich model (b).

The results of the corresponding parameters acquired by fitting the isothermal models were shown in Table 2. On the whole, a preferable fitting was gained with the Freundlich equation, showing that the adsorption process was non-monomolecular layer chemical adsorption. The DOX molecular may first enter into the specific cavities of MIP particles producing inductive fit with the MIP through hydrogen bonding formed by the amines of the MIP particles (APTES) and the carbonyl groups of the DOX. When all the accessible specific cavities of MIP particles were saturated, the adsorption of analyte was mainly due to nonspecific interactions, which could be the action mode for N-MIL on DOX adsorption.

Table 2. Isotherm parameters for DOX adsorption by MIP nanoparticles.

\begin{tabular}{ccccccc}
\hline & \multicolumn{3}{c}{ Langmuir } & \multicolumn{3}{c}{ Freundlich } \\
\cline { 2 - 7 } & $\boldsymbol{q}_{\mathrm{m}}(\mathbf{m g} / \mathbf{g})$ & $\boldsymbol{K}_{\mathrm{L}}(\mathbf{L} / \mathbf{m g})$ & $\boldsymbol{R}^{\mathbf{2}}$ & $\boldsymbol{K}_{\mathrm{F}}(\mathbf{L} / \mathbf{m g})$ & $\mathbf{1} / \boldsymbol{n}$ & $\boldsymbol{R}^{\mathbf{2}}$ \\
\hline N-MIL & 140.85 & 0.00332 & 0.7316 & 2.9060 & 0.5571 & 0.8415 \\
MIP & 5000 & 0.000669 & 0.7523 & 5.7227 & 0.8688 & 0.9877 \\
\hline
\end{tabular}

\subsection{In Vitro Drug Release}

Drug release rate analysis was used to evaluate the capability of MIP to deliver DOX, especially through sustainable release at two different $\mathrm{pH}(5.5,7.4)$, so as to simulate the human skin surface and body fluids environment, respectively. The results were compared with drug release from N-MIL particles. Figure 7 showed the $\mathrm{pH}$-responsive cumulative release profiles of MIP@DOX. It can be clearly seen that the drug release of MIP was slower and lower at $\mathrm{pH}$ 7.4. The initial rapid release of DOX was probably due to the weak adsorption of DOX molecules on the outer surface of the nanomaterials. It was noteworthy that N-MIL nanomaterials released a large quantity of DOX within $48 \mathrm{~h}$ (approximately $98 \%$ of DOX at $\mathrm{pH} 7.4$ ), while MIP only released $28.03 \%$ of drug within $72 \mathrm{~h}$ at $\mathrm{pH} 7.4$. Even at $\mathrm{pH} 5.5$, the cumulative release from MIP was eventually $46.19 \%$ after $72 \mathrm{~h}$. Consequently, although MIP and N-MIL both have sustained-release effects, the release of DOX from MIP nanomaterials was much slower and more delayed. The significant differences in the release of DOX from MIP nanoparticles and N-MIL nanoparticles can be owing to the presence of a specific site in the imprinted nanoparticles that strongly interacted with DOX molecules. 

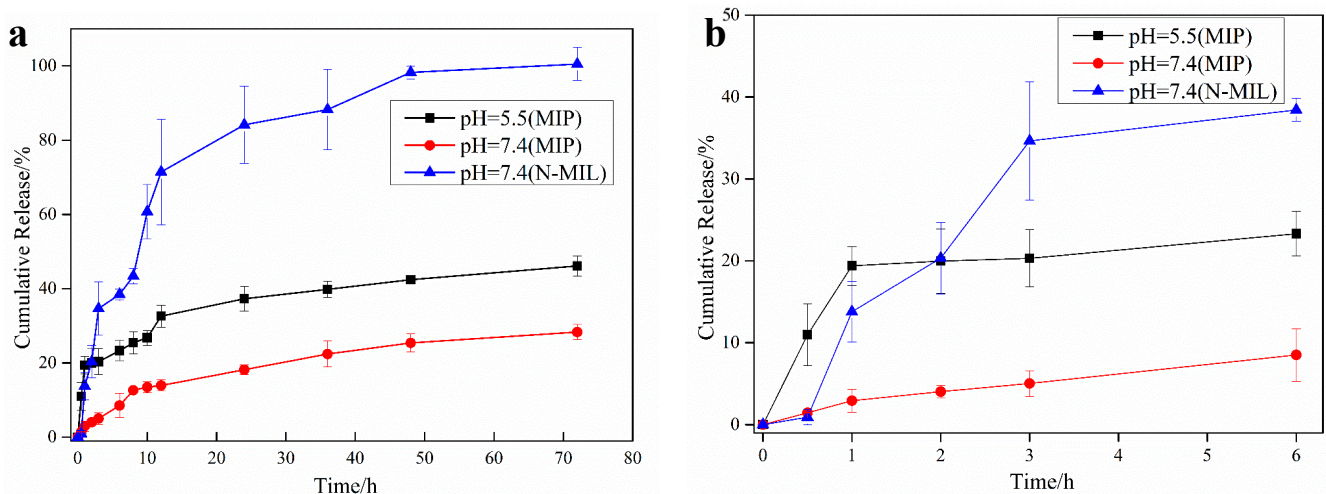

Figure 7. Cumulative percentage of DOX release from N-MIL@DOX and MIP@DOX in PBS (phosphate buffer saline) in $72 \mathrm{~h}(\mathbf{a})$ and over $6 \mathrm{~h}(\mathbf{b})$.

There are different models that have been applied to describe drug release kinetics from porous carriers, such as Korsmeyer-Peppas (Equation (5)) [34] and Sigmoidal model (Equation (6)) [35].

$$
\begin{gathered}
Q=K t^{n} \\
Q=\frac{R_{S}}{1+e^{-k_{s}\left(t-t_{50}\right)}}
\end{gathered}
$$

where $Q$ represents the cumulative release percentage at time $t(\mathrm{~h}), K$ is the kinetic constant, $n$ is diffusion exponent. The theoretical maximum release rate is $R_{S}(\%), k_{s}$ and $t_{50}$ are the release kinetic constant.

The results of the kinetic parameters for each model were summed up in Table 3. There was a noticeable distinction in the release kinetics of different nanomaterials under these conditions. Sigmoidal model cannot fit the experimental data well for MIP, while the Korsmeyer-Peppas model was more suitable for data fitting. However, for N-MIL system at pH 7.4, the experimental data did not conform well to the Korsmeyer-Peppas model, but the data had a good fit with the Sigmoidal model. According to the fitting results of Korsmeyer-Peppas in MIP sustained-release system in Table $3, \mathrm{n}$ was 0.26 at $\mathrm{pH} 5.5$ which was, less than 0.45 , revealing that the release kinetics process of the sustained-release system conformed to Fick diffusion mechanism. That was the interaction between MIP and drug was relatively weak. When $\mathrm{pH}$ was 7.4 , it was $0.45<\mathrm{n}<0.89$, demonstrating that the release kinetics of this system conformed to the non-Fick diffusion mechanism, and the interaction between MIP and the drug was obviously enhanced. Under different $\mathrm{pH}$ conditions, the sustained-release mechanism of MIP system changed, which was mainly due to the partial protonation of MIP at $\mathrm{pH}$ 5.5, which weakened the hydrogen bonding force between amino groups of MIP cavity and carbonyl groups of DOX. When $\mathrm{pH}$ was 7.4, the system was neutral, and the force between MIP and DOX increased. On the contrary, for the N-MIL release system, it was quickly released within the first $12 \mathrm{~h}$, and then released in an S shape until it reached the platform. The abnormal non-Fick spread is described by Sigmoidal release function. The change of drug diffusion and internal stresses resulted the abnormalities. The relaxation and diffusion time scales of macromolecular are similar in anomalous non-Fick diffusion [36-40].

Table 3. Correlation coefficient $\left(\mathrm{R}^{2}\right)$ of the release mechanism model Sigmoidal and Korsmeyer-Peppas according to various $\mathrm{pH}$.

\begin{tabular}{ccccccccc}
\hline \multirow{2}{*}{ Condition } & \multicolumn{3}{c}{ Korsmeyer-Peppas } & \multicolumn{4}{c}{ Sigmoidal Model } \\
\cline { 3 - 9 } & & $\mathbf{K}$ & $\mathbf{n}$ & $\mathbf{R}^{\mathbf{2}}$ & $\mathbf{k}_{\mathbf{s}}$ & $\mathbf{R}_{\mathbf{s}}$ & $\mathbf{t}_{50}$ & $\mathbf{R}^{\mathbf{2}}$ \\
\hline \multirow{2}{*}{ MIP } & $\mathrm{pH}=5.5$ & 15.7 & 0.26 & 0.95 & 0.18 & 41.7 & 4.89 & 0.85 \\
\multirow{2}{*}{ N-MIL } & $\mathrm{pH}=7.4$ & 2.63 & 0.59 & 0.97 & 0.20 & 24.1 & 9.55 & 0.91 \\
& $\mathrm{pH}=7.4$ & 7.89 & 0.26 & 0.76 & 0.27 & 93.1 & 7.68 & 0.95 \\
\hline
\end{tabular}




\subsection{Cytotoxicity Test}

To evaluate the potential application of the constructed MIP nanoparticles in DDS, the cytotoxicity tests of MIP and N-MIL on HepG2 cells and HL-7702 cells [41-46] were detected by MTT method (Figure 8). The cell viability showed that the inhibition rate increased with time increasing. Within $20 \mathrm{~h}$, the inhibition rates of MIP on HL-7702 cells and HepG2 cells were lower than 20\%, showing that MIP had no significant toxicity. Even after $24 \mathrm{~h}$, the rate of cell survival can still reach $76 \%$ and $70 \%$. However, after $24 \mathrm{~h}$ of incubation with the same concentration as MIP (20 $\mu \mathrm{g} / \mathrm{mL})$, MIP@DOX inhibited HepG2 and HL-7702 cells by $31 \%$ and $25 \%$, respectively, which were higher than that of MIP. The better inhibition rate of MIP@DOX on HepG2 was due to the slow drug release of DOX in this system. N-MIL and N-MIL@DOX systems also had the ability to inhibit HepG2 cells, but they can also kill normal liver HL-7702 cells at the same time. These results indicated that the toxicity of MIP to living cells was low in a certain time, and prove that the synthesized MIP has low toxicity and good biocompatibility.
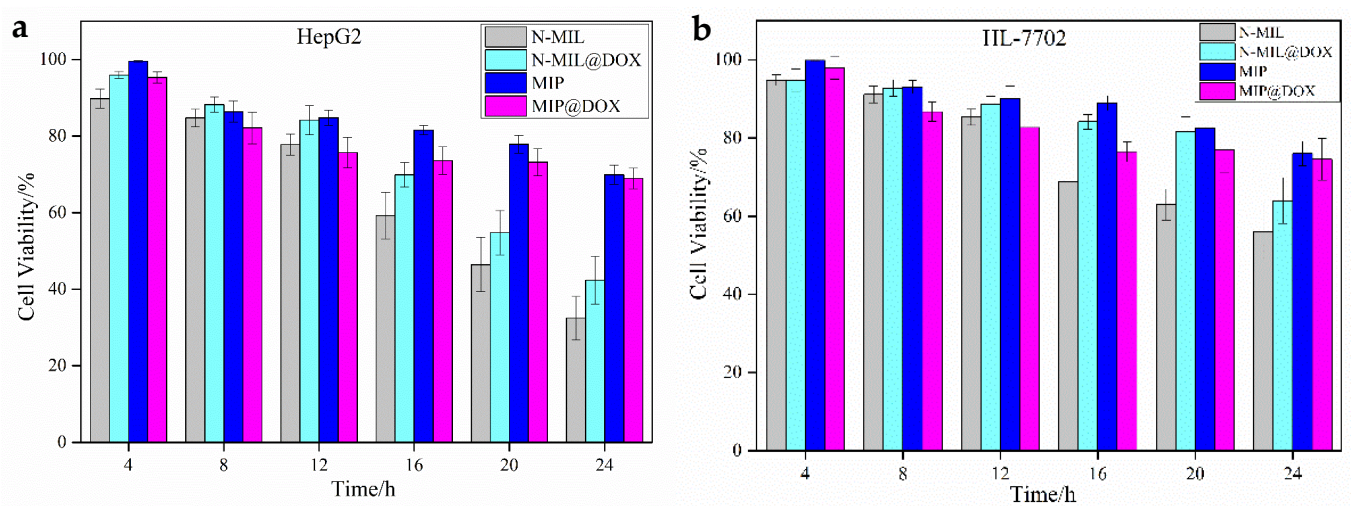

Figure 8. Cell viability of HepG2 cells (a) and HL-7702 cells (b) treated with N-MIL, N-MIL@DOX, MIP and MIP@DOX at different time.

To sum up, we synthesized a MIP with high drug loading efficiency, low toxicity and high biological activity. Before it is formally used as a clinical drug carrier, there may still be some restrictions for its application, such as the lack of in vivo experimental data support. Due to the poor water solubility of the synthesized MIP, which may be caused by the large size, it is not conducive to animal experiments. Therefore, our following work is to synthesize small-sized and water-soluble nanomaterials that can be clinically used for drug carriers. At present, this work is in progress.

\section{Conclusions}

In summary, we compounded MIL and N-CDs, and then successfully used the nanocomposite materials as framework, and DOX as template to synthesize MIP. Adsorption experiments showed that MIP had high sensitivity, specific selectivity and superior efficiency for DOX loading. It has a sustained release effect for DOX which cumulative release rates in $72 \mathrm{~h}$ were $46.19 \%$ and $28.03 \%$ at $\mathrm{pH} 5.5$ and 7.4, respectively. The cytotoxicity test results indicated that the inhibit rate of MIP on HepG2 and HL-7702 were $30.1 \%$ and $23.93 \%$, respectively, showing its good biological activity. In view of the outstanding biocompatibility, superior drug-loading and sustained releasing properties, this molecular imprinted polymer may have a potential to be used as a promising carrier in slow-released drug system in the future.

Author Contributions: Conceptualization, methodology, investigation, writing-original draft, Y.S.; investigation, writing—original draft, Y.W.; Project administration, Supervision, Writing—review and editing, J.Z.; Investigation, W.L.; Writing—review and editing, M.Z.H.K.; Supervision, X.L. All authors have read and agreed to the published version of the manuscript. 
Funding: This research was funded by National Science Foundation of China, grant number 21705033, the Medical Interdisciplinary Training Program of Henan University, grant number CJ1205A0240016 and Institute of Science and Technology of Henan University, grant number 2019YLZDYJ13.

Conflicts of Interest: The authors declare no conflict of interest. The funders had no role in the design of the study; in the collection, analyses, or interpretation of data; in the writing of the manuscript, or in the decision to publish the results.

\section{References}

1. Tian, J.; Ding, L.; Ju, H.; Yang, Y.; Li, X.; Shen, Z.; Zhu, Z.; Yu, J.S.; Yang, C.J. A multifunctional nanomicelle for real-time targeted imaging and precise near-infrared cancer therapy. Angew. Chem. Int. Ed. 2014, 53, 9544-9549. [CrossRef]

2. Siegel, R. Cancer statistics. CA Cancer J. Clin 2013, 63, 11-30. [CrossRef]

3. Khan, M.W.; Zhao, P.; Khan, A.; Raza, F.; Raza, S.M.; Sarfraz, M.; Chen, Y.; Li, M.; Yang, T.; Ma, X.; et al. Synergism of cisplatin-oleanolic acid co-loaded calcium carbonate nanoparticles on hepatocellular carcinoma cells for enhanced apoptosis and reduced hepatotoxicity. Int. J. Nanomed. 2019, 14, 3753. [CrossRef]

4. Tang, L.-Y.; Wang, Y.-C.; Li, Y.; Du, J.-Z.; Wang, J. Shell-detachable micelles based on disulfide-linked block copolymer as potential carrier for intracellular drug delivery. Bioconjugate Chem. 2009, 20, 1095-1099. [CrossRef]

5. Wang, F.; Wang, Y.-C.; Dou, S.; Xiong, M.-H.; Sun, T.-M.; Wang, J. Doxorubicin-tethered responsive gold nanoparticles facilitate intracellular drug delivery for overcoming multidrug resistance in cancer cells. ACS Nano 2011, 5, 3679-3692. [CrossRef]

6. Bullo, S.; Buskaran, K.; Fakurazi, S. Dual Drugs Anticancer Nanoformulation using Graphene Oxide-PEG as Nanocarrier for Protocatechuic Acid and Chlorogenic Acid. Pharm. Res. 2019, 36, 91. [CrossRef]

7. Zong, L.; Li, X.; Wang, H.; Cao, Y.; Yin, L.; Li, M.; Wei, Z.; Chen, D.; Pu, X. Formulation and characterization of biocompatible and stable IV itraconazole nanosuspensions stabilized by a new stabilizer polyethylene glycol-poly( $\beta$-Benzyl-L-aspartate) (PEG-PBLA). Int. J. Pharm. 2017, 531, 107-118. [CrossRef]

8. Zheng, H.; Zhang, Y.; Liu, L.; Wan, W.; Guo, P.; Nyström, A.M.; Zou, X. One-pot synthesis of metal-organic frameworks with encapsulated target molecules and their applications for controlled drug delivery. J. Am. Chem. Soc. 2016, 138, 962-968. [CrossRef]

9. Chowdhuri, A.R.; Singh, T.; Ghosh, S.K.; Sahu, S.K. Carbon dots embedded magnetic nanoparticles@ chitosan@ metal organic framework as a nanoprobe for $\mathrm{pH}$ sensitive targeted anticancer drug delivery. ACS Appl. Mater. Interfaces 2016, 8, 16573-16583. [CrossRef]

10. Qiu, B.; Shi, Y.; Yan, L.; Wu, X.; Zhu, J.; Zhao, D.; Khan, M.Z.H.; Liu, X. Development of an on-line immobilized $\alpha$-glucosidase microreactor coupled to liquid chromatography for screening of $\alpha$-glucosidase inhibitors. J. Pharm. Biomed. Anal. 2020, 180, 113047. [CrossRef]

11. Jiang, L.; Gao, Z.-M.; Ye, L.; Zhang, A.-Y.; Feng, Z.-G. A tumor-targeting nano doxorubicin delivery system built from amphiphilic polyrotaxane-based block copolymers. Polymer 2013, 54, 5188-5198. [CrossRef]

12. Li, L.; Chen, L.; Zhang, H.; Yang, Y.; Liu, X.; Chen, Y. Temperature and magnetism bi-responsive molecularly imprinted polymers: Preparation, adsorption mechanism and properties as drug delivery system for sustained release of 5-fluorouracil. Mater. Sci. Eng. C 2016, 61, 158-168. [CrossRef]

13. Li, Y.; Li, N.; Pan, W.; Yu, Z.; Yang, L.; Tang, B. Hollow mesoporous silica nanoparticles with tunable structures for controlled drug delivery. ACS Appl. Mater. Interfaces 2017, 9, 2123-2129. [CrossRef]

14. Demir, B.; Lemberger, M.M.; Panagiotopoulou, M.; Medina Rangel, P.X.; Timur, S.; Hirsch, T.; Tse Sum Bui, B.; Wegener, J.; Haupt, K. Tracking hyaluronan: Molecularly imprinted polymer coated carbon dots for cancer cell targeting and imaging. ACS Appl. Mater. Interfaces 2018, 10, 3305-3313. [CrossRef]

15. Liu, R.; Cui, Q.; Wang, C.; Wang, X.; Yang, Y.; Li, L. Preparation of sialic acid-imprinted fluorescent conjugated nanoparticles and their application for targeted cancer cell imaging. ACS Appl. Mater. Interfaces 2017, 9, 3006-3015. [CrossRef]

16. Zhang, W.; Kang, J.; Li, P.; Liu, L.; Wang, H.; Tang, B. Two-photon fluorescence imaging of sialylated glycans in vivo based on a sialic acid imprinted conjugated polymer nanoprobe. Chem. Commun. 2016, 52, 13991-13994. [CrossRef]

17. Ruela, A.L.M.; Figueiredo, E.C.; Pereira, G.R. Molecularly imprinted polymers as nicotine transdermal delivery systems. Biochem. Eng. J. 2014, 248, 1-8. [CrossRef] 
18. Pawley, C.J.; Perez-Gavilan, A.; Foley, K.S.; Lentink, S.; Welsh, H.N.; Tuijthof, G.; Steen Redeker, E.; Diliën, H.; Eersels, K.; Van Grinsven, B. Studying the Drug Delivery Kinetics of a Nanoporous Matrix Using a MIP-Based Thermal Sensing Platform. Polymers 2017, 9, 560. [CrossRef]

19. Wang, C.; Yang, K.; Wei, X.; Ding, S.; Tian, F.; Li, F. One-pot solvothermal synthesis of carbon dots/Ag nanoparticles/ $/ \mathrm{TiO}_{2}$ nanocomposites with enhanced photocatalytic performance. Ceram. Int. 2018, 44, 22481-22488. [CrossRef]

20. Qin, S.-J.; Yan, B. Dual-emissive ratiometric fluorescent probe based on Eu ${ }^{3+} / \mathrm{C}$-dots@ MOF hybrids for the biomarker diaminotoluene sensing. Sens. Actuators B 2018, 272, 510-517. [CrossRef]

21. Gu, C.; Guo, C.; Li, Z.; Wang, M.; Zhou, N.; He, L.; Zhang, Z.; Du, M. Bimetallic ZrHf-based metal-organic framework embedded with carbon dots: Ultra-sensitive platform for early diagnosis of HER2 and HER2-overexpressed living cancer cells. Biosens. Bioelectron. 2019, 134, 8-15. [CrossRef] [PubMed]

22. Chen, X.; Gao, H.; Yang, M.; Xing, L.; Dong, W.; Li, A.; Zheng, H.; Wang, G. Smart integration of carbon quantum dots in metal-organic frameworks for fluorescence-functionalized phase change materials. Energy Storage Mater. 2019, 18, 349-355. [CrossRef]

23. Niu, X.; Liu, G.; Li, L.; Fu, Z.; Xu, H.; Cui, F. Green and economical synthesis of nitrogen-doped carbon dots from vegetables for sensing and imaging applications. RSC Adv. 2015, 5, 95223-95229. [CrossRef]

24. Xie, Q.; Li, Y.; Lv, Z.; Zhou, H.; Yang, X.; Chen, J.; Guo, H. Effective adsorption and removal of phosphate from aqueous solutions and eutrophic water by Fe-based MOFs of MIL-101. Sci. Rep. 2017, 7, 1-15. [CrossRef] [PubMed]

25. Qian, K.; Deng, Q.; Fang, G.; Wang, J.; Pan, M.; Wang, S.; Pu, Y. Metal-organic frameworks supported surface-imprinted nanoparticles for the sensitive detection of metolcarb. Biosens. Bioelectron. 2016, 79, 359-363. [CrossRef] [PubMed]

26. Hamon, L.; Serre, C.; Devic, T.; Loiseau, T.; Millange, F.; Férey, G.r.; Weireld, G.D. Comparative study of hydrogen sulfide adsorption in the MIL-53 (Al, Cr, Fe), MIL-47 (V), MIL-100 (Cr), and MIL-101 (Cr) metalorganic frameworks at room temperature. J. Am. Chem. Soc. 2009, 131, 8775-8777. [CrossRef]

27. Peng, H.-H.; Hong, D.-X.; Guan, Y.-X.; Yao, S.-J. Preparation of pH-responsive DOX-loaded chitosan nanoparticles using supercritical assisted atomization with an enhanced mixer. Int. J. Pharm. 2019, 558, 82-90. [CrossRef]

28. Wang, H.-Y.; Cao, P.-P.; He, Z.-Y.; He, X.-W.; Li, W.-Y.; Li, Y.-H.; Zhang, Y.-K. Targeted imaging and targeted therapy of breast cancer cells via fluorescent double template-imprinted polymer coated silicon nanoparticles by an epitope approach. Nanoscale 2019, 11, 17018-17030. [CrossRef]

29. Qian, K.; Fang, G.; Wang, S. A novel core-shell molecularly imprinted polymer based on metal-organic frameworks as a matrix. Chem. Commun. 2011, 47, 10118-10120. [CrossRef]

30. Bene, L.; Bagdány, M.; Ungvári, T.; Damjanovich, L. Dual-Laser Tetra-Polarization FRET (4polFRET) for Site-Selective Control of Homo-FRET in Hetero-FRET Systems on the Cell Surface: The Homo-FRET Gate. Anal. Chem. 2018, 90, 10159-10170. [CrossRef]

31. Lovell, J.F.; Chan, M.W.; Qi, Q.; Chen, J.; Zheng, G. Porphyrin FRET acceptors for apoptosis induction and monitoring. J. Am. Chem. Soc. 2011, 133, 18580-18582. [CrossRef] [PubMed]

32. Santamaría, E.; Maestro, A.; Porras, M.; Gutiérrez, J.; González, C. Controlled release of ibuprofen by meso-macroporous silica. J. Solid State Chem. 2014, 210, 242-250. [CrossRef]

33. Freundlich, H. Of the adsorption of gases. Section II. Kinetics and energetics of gas adsorption. Introductory paper to section II. Trans. Faraday Soc. 1932, 28, 195-201. [CrossRef]

34. Korsmeyer, R.W.; Gurny, R.; Doelker, E.; Buri, P.; Peppas, N.A. Mechanisms of solute release from porous hydrophilic polymers. Int. J. Pharm. 1983, 15, 25-35. [CrossRef]

35. Narisawa, S.; Nagata, M.; Hirakawa, Y.; Kobayashi, M.; Yoshino, H. An organic acid-induced sigmoidal release system for oral controlled-release preparations. 2. Permeability enhancement of eudragit RS coating led by the physicochemical interactions with organic acid. J. Pharm. Sci. 1996, 85, 184-188. [CrossRef]

36. Ávila, M.I.; Alonso-Morales, N.; Baeza, J.A.; Rodriguez, J.J.; Gilarranz, M.A. High load drug release systems based on carbon porous nanocapsule carriers. Ibuprofen case study. J. Mater. Chem. B 2020, 8, 5293-5304. [CrossRef] 
37. Kim, A.R.; Lee, S.L.; Park, S.N. Properties and in vitro drug release of pH-and temperature-sensitive double cross-linked interpenetrating polymer network hydrogels based on hyaluronic acid/poly (N-isopropylacrylamide) for transdermal delivery of luteolin. Int. J. Biol. Macromol. 2018, 118, 731-740. [CrossRef]

38. Saxena, A.; Kaloti, M.; Bohidar, H. Rheological properties of binary and ternary protein-polysaccharide co-hydrogels and comparative release kinetics of salbutamol sulphate from their matrices. Int. J. Biol. Macromol. 2011, 48, 263-270. [CrossRef]

39. Wang, Y.; Yang, M.; Shen, R.; Shao, S.; Chen, L.; Gong, W.; Shan, L.; Gao, C. Development of metoprolol tartrate-loaded sustained-release pellets: Effect of talc on the mechanism of drug release. Pharm. Dev. Technol. 2018, 23, 664-673. [CrossRef]

40. Shi, Y.; Qiu, B.; Wu, X.; Wang, Y.; Zhu, J.; Liu, X.; Zhao, D. Drug delivery system and in vitro release of luteolin based on magnetic nanocomposite $\left(\mathrm{Fe}_{3} \mathrm{O}_{4} @ \mathrm{ZIF}-67\right)$. Micro Nano Lett. 2020, 15, 425-429.

41. Yang, Y.; Yu, B.; Zhao, T.; Xu, S.; Zhang, H. Cytotoxicity of gold nanoclusters in human liver cancer cells. Int. J. Nanomed. 2014, 9, 5441-5448. [CrossRef] [PubMed]

42. Huang, S.; Wang, W.; Li, H.; Hu, Y. A 54 peptide-mediated functionalized gold nanocages for targeted delivery of DOX as a combinational photothermal-chemotherapy for liver cancer. Int. J. Nanomed. 2017, 12, 5163-5176. [CrossRef] [PubMed]

43. Ji, M.; Hou, L.; Huang, S.; Li, Y.; Liu, Y.; Hu, Y. Construction and application of a liver cancer-targeting drug delivery system based on core-shell gold nanocages. Int. J. Nanomed. 2018, 13, 1773-1789. [CrossRef] [PubMed]

44. Sun, Z.; Huang, G. Synthesis of theranostic Anti-EGFR ligand conjugate iron oxide nanoparticles for magnetic resonance imaging for treatment of liver cancer. J. Drug Delivery Sci. Technol. 2020, 55, 101367. [CrossRef]

45. Du, L.; Wu, L.; Jin, Y.; Jia, J.; Li, M.; Wang, Y. Self-assembled drug delivery systems. Part 7: Hepatocyte-targeted nanoassemblies of an adefovir lipid derivative with cytochrome P-450-triggered drug release. Int. J. Pharm. 2014, 472, 1-9. [CrossRef] [PubMed]

46. Wang, W.; Huang, S.; Yuan, J.; Xu, X.; Li, H.; Lv, Z.; Yu, W.; Hu, Y. Reverse Multidrug Resistance in Human HepG2/ADR by Anti-miR-21 Combined with Hyperthermia Mediated by Functionalized Gold Nanocages. Mol. Pharm. 2018, 15, 3767-3776. [CrossRef]

(C) 2020 by the authors. Licensee MDPI, Basel, Switzerland. This article is an open access article distributed under the terms and conditions of the Creative Commons Attribution (CC BY) license (http://creativecommons.org/licenses/by/4.0/). 